TUW-98-09

\title{
Global properties of warped solutions in General Relativity
}

\author{
M. O. Katanaev*, T. Klösch ${ }^{\dagger}$, W. Kummer ${ }^{\ddagger}$ \\ *Steklov Mathematical Institute \\ Gubkin St. 8, Moscow 117966, Russia \\ †Institut für Theoretische Physik \\ Technische Universität Wien \\ Wiedner Hauptstr. 8-10, A-1040 Wien, Austria
}

\begin{abstract}
Assuming the four-dimensional space-time to be a general warped product of two surfaces we reduce the four-dimensional Einstein equations to a two-dimensional problem which can be solved. All global vacuum solutions are explicitly constructed and analysed. The classification of the solutions includes the Schwarzschild, the (anti-)de Sitter, and other well-known solutions but also many exact ones whose detailed global properties to our knowledge have not been discussed before. They have a natural physical interpretation describing single or several wormholes, domain walls of curvature singularities, cosmic strings, cosmic strings surrounded by domain walls, solutions with closed timelike curves, etc.
\end{abstract}

November 11, 1998

\footnotetext{
${ }^{*}$ E-mail: katanaev@mi.ras.ru

${ }^{\dagger}$ E-mail: kloesch@tph.tuwien.ac.at

${ }_{\ddagger}^{\ddagger}$ E-mail: wkummer@tph.tuwien.ac.at
} 


\section{Introduction}

Since Einstein's discovery of general relativity the search for and the study of exact solutions has been a field of continuous activity. As a consequence the literature on the subject is huge. Several examples of systematic approaches are described in [1]. It is a common feature of all exact solutions that special symmetries are assumed which lead to Killing fields on the corresponding manifolds. The venerable Schwarzschild solution with a spherical symmetry is the simplest example, corresponding to an effective $2 d$ theory. It may be derived by spherically symmetric reduction from the Einstein-Hilbert action of general relativity. A $1+1$ metric and a dilaton field are its effective variables [2].

The increasing interest in string theories also stimulated studies of $2 d$ covariant theories. Generalizing the (trivial) $2 d$ Einstein gravity from string theory, e.g., led to gravity theory in $1+1$ dimensions with nonvanishing torsion [3]. String theory also inspired the dilaton black hole [四, whose Lagrangian closely resembles the one of spherically reduced general relativity. Although its global solution is described by the same Carter-Penrose diagram as the one for the Schwarzschild black hole, the singularity is different, admitting complete null geodesics [5]. Within the past decade also generalized dilaton theories have been studied widely [6] and, e.g., their usefulness for modelling (even globally) the Schwarzschild black hole was shown [7]. Important progress has been made in the nonperturbative quantum treatment of such theories where the use of a "lightcone" gauge for the Cartan variables leads to an Eddington-Finkelstein gauge for the metric. This allows for an exact path integral in the geometric variables [8].

Most of the considered $2 d$ gravity models are simple enough to be integrable. This raises the fundamental question of how to construct the corresponding maximally extended surfaces or global solutions. By this we mean that any extremal (or geodesic) can be continued to an infinite value of its canonical parameter in both directions unless it runs into a curvature singularity at some finite distance. This is an important issue because almost all coordinate representations of metrics are not maximally extended, indicating that they describe only a small part of a larger solution. A well-known example is the Schwarzschild solution, the maximal extension of which can be covered by Kruskal-Szekeres coordinates [9].

The Carter-Penrose diagrams for the most important Schwarzschild, ReissnerNordstrom, and Kerr solutions were constructed by Carter and discuseed in his seminal review [10]. His method is based on the explicit construction of overlapping coordinate systems. It is applicable only to metrics of Lorentz signature and thus the universality of the method is unclear. A systematic and equivalent method for the construction of maximally extended solutions was proposed in [11] for $2 d$ gravity with torsion in the conformal gauge. It is based on the analysis of extremals and does not use overlapping coordinate systems. The disadvantage of the approach is that the continuity of the metric was proved only up to second order on the horizons. A more general approach based on a Poisson-sigma-model 
formulation of generalized dilaton-type models and $2 d$ gravity with torsion has been developed in [12]. It uses overlapping coordinate systems and proves that the maximally extended solutions are smooth. These rules may be used for a large class of $2 d$ metrics without explicit construction of Kruskal-Szekeres-like coordinate systems and without concern for smoothness of the resulting maximally extended solution; hence it has been proved in a general case. A systematic construction of the maximally extended solutions for the Euclidean signature metrics was proposed in [11].

Starting from Einstein's gravity in four dimensions, we make a special ansatz for a metric reducing the problem to a two-dimensional one, and then construct all corresponding maximally extended solutions. We assume that the fourdimensional space-time manifold $\mathbb{M}$ with local coordinates $x^{i}(i, j, \ldots=0,1,2,3)$ is a warped product of two surfaces $\mathbb{M}=\mathbb{U} \times \mathbb{V}$. This means that in a suitable coordinate system $x^{i}=\left\{x^{\alpha}, y^{\mu}\right\},(\alpha, \beta, \ldots=0,1),(\mu, \nu, \ldots=2,3)$, where $x^{\alpha}$ and $y^{\mu}$ are coordinates on the surfaces $\mathbb{U}$ and $\mathbb{V}$, respectively, the four-dimensional metric has a block diagonal form

$$
\widehat{g}_{i j}=\left(\begin{array}{cc}
k(y) g_{\alpha \beta}(x) & 0 \\
0 & m(x) h_{\mu \nu}(y)
\end{array}\right) .
$$

Here and in what follows we use the hat symbol to denote geometrical quantities which refer to the whole spacetime $\mathbb{M}$, whereas symbols without hats are used for $2 d$ surfaces. Accordingly, $g_{\alpha \beta}$ and $h_{\mu \nu}$ are metric tensors and $m(x)$ and $k(y)$ are scalar (dilaton) fields on the surfaces $\mathbb{U}$ and $\mathbb{V}$, respectively. Greek letters from the beginning $(\alpha, \beta, \ldots)$ and the middle $(\mu, \nu, \ldots)$ of the alphabet always refer to coordinates on the first and the second surface.

For definiteness we specify $\mathbb{U}$ to be a pseudo-Riemannian manifold with a Lorentz signature metric and $\mathbb{V}$ a Riemannian manifold with positive or negative definite metric. Then up to a permutation of the first two coordinates the signature of the metric on $\mathbb{M}$ will be either $(+---)$ or $(-+++)$ depending on the sign of $m$. These solutions are related to each other by an inversion of the metric $g_{i j} \rightarrow-g_{i j}$ which is a symmetry transformation of the Einstein equations in the absence of matter fields. Both surfaces are assumed to be orientable. Note that we do not assume initially any symmetry of the metric (11). Only as the consequence of Einstein's equations and the requirement of completeness we find that any solution of the form (11) must be highly symmetric

Many well-known metrics of general relativity have this form. For example, for the Schwarzschild solution the surface $\mathbb{V}$ is a $2 d$ sphere $\mathbb{S}^{2}$ and $\mathbb{U}$ is represented by the Carter-Penrose diagram for a black hole [10]. For some recent work on warped metrics cf. [14].

As far as their local properties are concerned all solutions discussed below are well known and belong to type $D$ according to Petrov's classification [15. Also the global structure of spherically symmetric solutions has been discussed already in [10]. The global structure of the planar and Lobachevsky plane solutions 
were described quite recently [16]. The global structure of the hyperbolic and Minkowski plane solutions constructed in the present paper is new. The physical interpretation relies on the global structure. We find that the solutions describe domain walls of curvature singularities, cosmic strings surrounded by domain walls, and others. In this construction we use the teqnique of [13].

The metric (1) allows us to solve explicitly the four-dimensional vacuum Einstein equations with a cosmological constant $\Lambda$

$$
\widehat{R}_{i j}=\Lambda \widehat{g}_{i j}
$$

and to construct the possible (maximally extended) global solutions. In Section 2 we perform the reduction of Einstein's equations for the metric (11). The Einstein equations severely restrict the dilaton fields; in fact, at least one of them must be constant. Hence solutions are grouped into three main classes, corresponding to the dilaton fields being both constant (case $A$ ), only $k=$ const (case $B$ ), or only $m=$ const (case $C$ ). As shown in Section 3, case $A$ leads to solutions of a rather simple type (product of two constant curvature surfaces). Spatially symmetric solutions (case $B$ ) are the subject of Section 4 . Here the well-known spherically and planar solutions are rederived. However, also a class of solutions which are warped products of some pseudo-Riemanian surface with a Lobachevsky plane or with a higher genus Riemanian surface having $\mathrm{O}(1,2)$ as symmetry group appear. They are physically interpreted as $n$ wormhole solutions, where $n$ is the number of handles on the corresponding Riemann surface. It should be noted that in this connection that planar and Lobachevsky plane solutions of black hole type recently attracted growing interest [16, 17]. In Section 5 for the case $C$ ( $m=$ const) we have constructed new vacuum solutions to the Einstein equations which seem to be unknown at the global level. Again their global properties are discussed in detail. The physical interpretation of these solutions is quite interesting. They describe cosmic strings and domain walls of curvature singularities. In Section 6 we summarize our results.

\section{Two-dimensional reduction}

The inverse metric to (11) is

$$
\widehat{g}^{i j}=\left(\begin{array}{cc}
\frac{1}{k} g^{\alpha \beta} & 0 \\
0 & \frac{1}{m} h^{\mu \nu}
\end{array}\right)
$$


where $g^{\alpha \beta}$ and $h^{\mu \nu}$ are inverse matrices to $g_{\alpha \beta}$ and $h_{\mu \nu}$. The components of the Christoffel symbols become

$$
\begin{aligned}
\widehat{\Gamma}_{\alpha \beta}^{\gamma} & =\Gamma_{\alpha \beta}{ }^{\gamma}, \\
\widehat{\Gamma}_{\alpha \beta}{ }^{\mu} & =-\frac{1}{2} g_{\alpha \beta} \frac{h^{\mu \nu} \partial_{\nu} k}{m}, \\
\widehat{\Gamma}_{\alpha \mu}^{\gamma} & =\widehat{\Gamma}_{\mu \alpha}^{\gamma}=\frac{1}{2} \delta_{\alpha}^{\gamma} \frac{\partial_{\mu} k}{k} \\
\widehat{\Gamma}_{\alpha \mu}{ }^{\nu} & =\widehat{\Gamma}_{\mu \alpha}{ }^{\nu}=\frac{1}{2} \delta_{\mu}^{\nu} \frac{\partial_{\alpha} m}{m} \\
\widehat{\Gamma}_{\mu \nu}{ }^{\alpha} & =-\frac{1}{2} h_{\mu \nu} \frac{g^{\alpha \beta} \partial_{\beta} m}{k} \\
\widehat{\Gamma}_{\mu \nu}{ }^{\rho} & =\Gamma_{\mu \nu}{ }^{\rho} .
\end{aligned}
$$

With the definition of the curvature tensor

$$
\widehat{R}_{i j k}^{l}=\partial_{i} \widehat{\Gamma}_{j k}^{l}-\widehat{\Gamma}_{i k}^{m} \widehat{\Gamma}_{j m}^{l}-(i \leftrightarrow j),
$$

the components of the Ricci tensor, $\widehat{R}_{i j}=\widehat{R}_{i k j}{ }^{k}$, are

$$
\begin{aligned}
\widehat{R}_{\alpha \beta} & =R_{\alpha \beta}+\frac{\nabla_{\alpha} \nabla_{\beta} m}{m}-\frac{\nabla_{\alpha} m \nabla_{\beta} m}{2 m^{2}}+\frac{g_{\alpha \beta} \nabla^{2} k}{2 m} \\
\widehat{R}_{\alpha \mu} & =\widehat{R}_{\mu \alpha}=-\frac{\nabla_{\alpha} m \nabla_{\mu} k}{2 m k} \\
\widehat{R}_{\mu \nu} & =R_{\mu \nu}+\frac{\nabla_{\mu} \nabla_{\nu} k}{k}-\frac{\nabla_{\mu} k \nabla_{\nu} k}{2 k^{2}}+\frac{h_{\mu \nu} \nabla^{2} m}{2 k}
\end{aligned}
$$

where

$$
\nabla^{2} m=g^{\alpha \beta} \nabla_{\alpha} \nabla_{\beta} m, \quad \nabla^{2} k=h^{\mu \nu} \nabla_{\mu} \nabla_{\nu} k
$$

The covariant derivative acting on the scalar fields $m, k$ coincides with the partial derivative while it acts on vectors on $\mathbb{U}$ as

$$
\nabla_{\alpha} V^{\beta}=\partial_{\alpha} V^{\beta}+\Gamma_{\alpha \gamma}^{\beta} V^{\gamma}
$$

and analogously in $\mathbb{V}$. Now the scalar curvature, $\widehat{R}=\widehat{R}_{i}{ }^{i}$, becomes

$$
\widehat{R}=\frac{1}{k} R^{g}+2 \frac{\nabla^{2} m}{k m}-\frac{(\nabla m)^{2}}{2 k m^{2}}+\frac{1}{m} R^{h}+2 \frac{\nabla^{2} k}{k m}-\frac{(\nabla k)^{2}}{2 k^{2} m},
$$

with the obvious shorthand notations

$$
(\nabla m)^{2}=g^{\alpha \beta} \partial_{\alpha} m \partial_{\beta} m, \quad(\nabla k)^{2}=h^{\mu \nu} \partial_{\mu} k \partial_{\nu} k .
$$

$R^{g}$ and $R^{h}$ are scalar curvatures of the surfaces $\mathbb{U}$ and $\mathbb{V}$, respectively. 
63 SOLUTIONS WITH CONSTANT CURVATURE SURFACES (CASE A).

Thus the Einstein equations (2) for the metric (11) reduce to

$$
\begin{aligned}
R_{\alpha \beta}+\frac{\nabla_{\alpha} \nabla_{\beta} m}{m}-\frac{\nabla_{\alpha} m \nabla_{\beta} m}{2 m^{2}}+\frac{1}{2} g_{\alpha \beta}\left(\frac{\nabla^{2} k}{m}-2 k \Lambda\right) & =0, \\
R_{\mu \nu}+\frac{\nabla_{\mu} \nabla_{\nu} k}{k}-\frac{\nabla_{\mu} k \nabla_{\nu} k}{2 k^{2}}+\frac{1}{2} h_{\mu \nu}\left(\frac{\nabla^{2} m}{m}-2 m \Lambda\right) & =0, \\
\frac{\nabla_{\alpha} m \nabla_{\mu} k}{m k} & =0 .
\end{aligned}
$$

Equations (18), (19) are rewritten in a more suitable form by extracting the traces which yield scalar curvatures for the surfaces

$$
\begin{aligned}
& R^{g}+\frac{\nabla^{2} m}{m}-\frac{(\nabla m)^{2}}{2 m^{2}}+\frac{\nabla^{2} k}{m}-2 k \Lambda=0 \\
& R^{h}+\frac{\nabla^{2} k}{k}-\frac{(\nabla k)^{2}}{2 k^{2}}+\frac{\nabla^{2} m}{k}-2 m \Lambda=0 .
\end{aligned}
$$

The traceless parts of eqs. (18), (19) multiplied by $m$ and $k$ take the simple form

$$
\begin{aligned}
\nabla_{\alpha} \nabla_{\beta} m-\frac{\nabla_{\alpha} m \nabla_{\beta} m}{2 m}-\frac{1}{2} g_{\alpha \beta}\left[\nabla^{2} m-\frac{(\nabla m)^{2}}{2 m}\right] & =0, \\
\nabla_{\mu} \nabla_{\nu} k-\frac{\nabla_{\mu} k \nabla_{\nu} k}{2 k}-\frac{1}{2} h_{\mu \nu}\left[\nabla^{2} k-\frac{(\nabla k)^{2}}{2 k}\right] & =0 .
\end{aligned}
$$

They do not contain curvature terms at all because in two dimensions the Ricci tensor is completely defined by the scalar curvature,

$$
R_{\alpha \beta}=\frac{1}{2} g_{\alpha \beta} R^{g},
$$

and has no traceless part. Note that for a surface $\mathbb{U}$ the absence of a singularity in the scalar curvature implies its absence in the full curvature tensor.

Thus the four-dimensional vacuum Einstein equations (2) for a metric of the form (11) are equivalent to a system of equations (20)-(24). Equations (23) and (24) contain functions which depend only on $x$ and $y$, respectively, while coordinates in the other equations (20), (21), and (22) are mixed. Equation (20) is very restrictive. It states that either $k$ or $m$ or both $k$ and $m$ are constant:

$$
\begin{array}{rrr}
A: & k=\text { const } \neq 0, \quad m=\text { const } \neq 0, \\
B: & k=\text { const } \neq 0, & \nabla_{\alpha} m \neq 0, \\
C: & \nabla_{\mu} k \neq 0, & m=\text { const } \neq 0 .
\end{array}
$$

\section{Solutions with constant curvature surfaces (case A).}

The most symmetric warped product solutions to the reduced Einstein equations are obtained when both $k$ and $m$ are constants. In this case equations (20), (23), 
and (24) are satisfied and the scalar curvatures of both surfaces $\mathbb{U}$ and $\mathbb{V}$ are constant as a consequence of eqs. (21), (22) which reduce to

$$
\begin{aligned}
& R^{g}=2 k \Lambda, \\
& R^{h}=2 m \Lambda .
\end{aligned}
$$

If $\Lambda=0$ then both $\mathbb{U}$ and $\mathbb{V}$ are surfaces of zero curvature and $\mathbb{M}$ is Minkowski space or a compactified version (cylinder, torus) with the metric

$$
\widehat{g}_{i j}=\operatorname{diag}(+---) \quad \text { or } \quad \widehat{g}_{i j}=\operatorname{diag}(-+++) .
$$

For nonzero $\Lambda$ both surfaces $\mathbb{U}$ and $\mathbb{V}$ are of constant nonzero curvature. These surfaces are well-known so we give here only explicit expressions for the corresponding metrics. If $\mathbb{U}$ is a complete pseudo-Riemannian manifold of nonzero constant curvature, $R^{g}=-2 K=$ const, then it may be represented as the one sheet hyperboloid, $\mathbb{H}^{2}$, imbedded in three-dimensional Minkowski space with the induced metric or its universal covering space (cf. e.g. [11]). Its symmetry group is the orthogonal group $O(1,2)$. In stereographic coordinates its metric has the standard form

$$
d \Phi^{2}=g_{\alpha \beta} d x^{\alpha} d x^{\beta}=\frac{d t^{2}-d x^{2}}{\left[1+\frac{K}{4}\left(t^{2}-x^{2}\right)\right]^{2}},
$$

where we have denoted $t=x^{0}$ and $x=x^{1}$ in order to give the metric a more familiar appearance. In contrast to the Riemannian case the surface is now the same for positive and negative scalar curvature $K$ but the metric (29) changes its sign corresponding to permutation of the coordinates $t \leftrightarrow x$. For $K=0$ the metric (29) is the usual $2 d$ Minkowskian metric, and the corresponding surface is a Minkowskian plane, $\mathbb{M}^{2}$, with the inhomogeneous group $I O(1,1)$ as symmetry group, resp. a cylinder or a torus.

The positive definite metric for a $2 d$ Riemannian manifold of constant curvature, $R^{h}=-2 K$, in stereographic coordinates has the form

$$
d \Omega^{2}=h_{\mu \nu} d y^{\mu} d y^{\nu}=\frac{d y^{2}+d z^{2}}{\left[1+\frac{K}{4}\left(y^{2}+z^{2}\right)\right]^{2}},
$$

where $y=y^{1}$ and $z=y^{2}$. This metric differs from (29) only in the signs. For positive $K>0$ it corresponds to a sphere $\mathbb{S}^{2}$. With our definition of the curvature and Ricci tensors the scalar curvature of a sphere with positive definite metric is negative. For $K=0$ the metric (30) describes the Euclidean plane $\mathbb{R}^{2}$, or a cylinder, or a torus. For negative $K<0$ one has the Lobachevsky (or hyperbolic) plane $\mathbb{L}^{2}$ or (after compactification) some higher genus Riemannian surface. The symmetry groups for a sphere, Euclidean and Lobachevsky planes are $O(3)$, $I O(2)$, and $O(1,2)$, respectively. For positive and negative scalar curvatures one can always rescale coordinates in such a way that $K= \pm 1$. 
If the scalar curvatures are constant as in (26), (27) then the most symmetric solution for nonzero $\Lambda$ which is the warped product of two surfaces has the form

$$
d s^{2}=k \frac{d t^{2}-d x^{2}}{\left[1-\frac{k \Lambda}{4}\left(t^{2}-x^{2}\right)\right]^{2}}+m \frac{d y^{2}+d z^{2}}{\left[1-\frac{m \Lambda}{4}\left(y^{2}+z^{2}\right)\right]^{2}} .
$$

Rescaling the coordinates one may set $k= \pm 1, m= \pm 1$. We choose $k=1$ and $m=-1$ in order for the metric to have the signature $(+---)$. Then there are three essentially different solutions corresponding to positive, zero, and negative cosmological constant:

$$
\begin{array}{llll}
\Lambda<0 & R^{g}=-2|\Lambda| & R^{h}=+2|\Lambda| & \mathbb{M}=\mathbb{H}^{2} \times \mathbb{L}^{2} \\
\Lambda=0 & R^{g}=0 & R^{h}=0 & \mathbb{M}=\mathbb{M}^{2} \times \mathbb{R}^{2} \\
\Lambda>0 & R^{g}=+2|\Lambda| & R^{h}=-2|\Lambda| & \mathbb{M}=\mathbb{H}^{2} \times \mathbb{S}^{2}
\end{array}
$$

Although the scalar curvature for all these manifolds (as of course for any solution of Einstein's equations (2)) is constant, $\widehat{R}=4 \Lambda$, they do not coincide with the corresponding de Sitter solutions: each of the surfaces $\mathbb{M}^{2}, \mathbb{H}^{2}, \mathbb{L}^{2}, \mathbb{R}^{2}$, and $\mathbb{S}^{2}$ has three Killing vector fields, and the whole space-time possesses six, whereas the de Sitter solution has ten Killing vector fields. The above solutions are known (see, for example, [1]) and belong to the $D$ type according to Petrov's classification [15.

\section{Spatially symmetric solutions (case $B$ ).}

The case $B$ with $k=1$ describes spatially symmetric solutions with the symmetry group $O(3), I O(2)$, or $O(1,2)$ for positive, zero, or negative scalar curvature of $\mathbb{V}$, respectively. Here we rederive the well-known spherically, planar and Lobachevsky plane solutions. The global space-time is a warped product of $\mathbb{U}$ with $\mathbb{S}^{2}, \mathbb{R}^{2}$, or $\mathbb{L}^{2}$, where $\mathbb{U}$ is represented by a Carter-Penrose diagram. Although these solutions are well known we review them in detail to make the article self contained, to formulate the general rules of global construction, and to compare those solutions with the hyperbolic ones.

For $k=1$ the whole set of Einstein equations (20)-(24) reduces to

$$
\begin{aligned}
\nabla_{\alpha} \nabla_{\beta} m-\frac{\nabla_{\alpha} m \nabla_{\beta} m}{2 m}-\frac{1}{2} g_{\alpha \beta}\left[\nabla^{2} m-\frac{(\nabla m)^{2}}{2 m}\right] & =0, \\
R^{h}+\nabla^{2} m-2 m \Lambda & =0, \\
R^{g}+\frac{\nabla^{2} m}{m}-\frac{(\nabla m)^{2}}{2 m^{2}}-2 \Lambda & =0 .
\end{aligned}
$$

Equation (34) implies that the sum of two functions depending on different coordinates equals zero. Therefore each of the functions must be a constant. Let 
us fix that constant as $R^{h}=-2 K=$ const. Then eq. (34) is replaced by

$$
\nabla^{2} m-2(m \Lambda+K)=0 .
$$

Excluding the case $A$ of the previous section we proceed requiring $\nabla_{\alpha} m \neq 0$. Then (36) is a first integral to eqs. (33), (35). To prove this one has to differentiate equation (36), to use the identity

$$
\left[\nabla_{\alpha}, \nabla_{\beta}\right] V_{\gamma}=-R_{\alpha \beta \gamma}^{g}{ }^{\delta} V_{\delta},
$$

to exchange the order of covariant derivatives, and to use (33) three times to eliminate second derivatives of $m$. After some algebra one finally obtains equation (35). Thus only equations (33) and (36) must be solved, (35) being satisfied automatically.

We now choose a conformal gauge on $\mathbb{U}$,

$$
g_{\alpha \beta} d x^{\alpha} d x^{\beta}=2 g d u d v=2 g\left(d \tau^{2}-d \sigma^{2}\right),
$$

where $g(u, v)$ is a function of two light-cone coordinates on $\mathbb{U}$,

$$
u=\tau+\sigma, \quad v=\tau-\sigma .
$$

Here we denote the conformal coordinates by Greek letters because later the transformation to Schwarzschild coordinates will require functions $r=r(\sigma)$ and $t=t(\tau)$. The four-dimensional line element takes the form

$$
d s^{2}=2 g d u d v+m d \Omega^{2} .
$$

Without loss of generality we assume here that $g>0$. Otherwise one merely has to exchange the first two coordinates.

The Christoffel symbols for the metric (37) in conformal gauge have only two nonvanishing components

$$
\Gamma_{u u}^{u}=\frac{\partial_{u} g}{g}, \quad \Gamma_{v v}^{v}=\frac{\partial_{v} g}{g},
$$

and (33), (36) take the simple form

$$
\begin{aligned}
\partial_{u} \partial_{u} m-\frac{\partial_{u} m \partial_{u} m}{2 m}-\frac{\partial_{u} g \partial_{u} m}{g} & =0, \\
\partial_{v} \partial_{v} m-\frac{\partial_{v} m \partial_{v} m}{2 m}-\frac{\partial_{v} g \partial_{v} m}{g} & =0, \\
\frac{2 \partial_{u} \partial_{v} m}{g}-2(m \Lambda+K) & =0 .
\end{aligned}
$$

Thus the full set of eqs. (33)-(35) in the conformal gauge (37) reduces to three equations for two unknown functions $m$ and $g$. This system of equations is 
overdefined and can be integrated explicitly. The first two are ordinary differential equations and coincide with the equations of $2 d$ gravity with torsion 18 . Therefore we only sketch their integration. Dividing them by $\partial_{u} m$ and $\partial_{v} m$, respectively, they can be integrated easily. There arise two arbitrary functions corresponding to the invariance of (41)-(43) under the conformal transformations $u \rightarrow u^{\prime}(u)$ and $v \rightarrow v^{\prime}(v)$. They may be chosen in such a way that the functions $m(u \pm v)$ and $g(u \pm v)$ depend simultaneously on either a time-like or a space-like coordinate

$$
\zeta=\frac{1}{2}(u \pm v)=\tau \text { or } \sigma .
$$

This means that the metric has a Killing vector field as the consequence of equations (41) and (42). We call these solutions homogeneous and static, respectively, although this refers only to a specified coordinate system. This is a generalization of the Birkhoff's theorem stating that a spherically symmetric solution of the Einstein equations must be static. The second and the last consequence of eqs. (41) and (42) is the expression for $g$ in terms of $m$,

$$
g=\frac{\left|m^{\prime}\right|}{4 \sqrt{|m|}},
$$

where the prime denotes the derivative with respect to $\zeta$ defined in (44). Here we have used the assumption $g>0$ and for later convenience introduced a factor $1 / 4$. We are free to do this because equations (41) and (42) define $g$ only up to an arbitrary factor. Inserting expression (45) into the last equation (43) we arrive at the ordinary differential equation determining $m$,

$$
\pm m^{\prime \prime}-(m \Lambda+K) \frac{\left|m^{\prime}\right|}{\sqrt{|m|}}=0
$$

where the upper and lower signs, \pm , correspond to homogeneous and static solutions, respectively. Its integral depends on the sign of $m$,

$$
\left|\frac{d m}{d \zeta}\right|=\mp 2 W(m),
$$

where

$$
\begin{array}{lll}
W(m)=-\frac{1}{3} \Lambda m^{3 / 2}-K m^{1 / 2}-2 M, & & m>0, \\
W(m)=-\frac{1}{3} \Lambda(-m)^{3 / 2}+K(-m)^{1 / 2}-2 M, & & m<0 .
\end{array}
$$

Here $M$ is an arbitrary integration constant which will be seen to coincide with the mass in the Schwarzschild solution. The equation for positive and negative $m$ differs only in the sign of $K$. 
The line element of the solution in conformal gauge is thus

$$
d s^{2}=\mp \frac{W(m)}{|m|^{1 / 2}}\left(d \tau^{2}-d \sigma^{2}\right)-m d \Omega^{2},
$$

where $m$ depends either on $\tau$ or $\sigma$ through equation (47). The sign is obtained from equation (47): The left-hand side of the equation is always positive and the same must be true for the right-hand side. At the same time when $m$ varies from $-\infty$ to $+\infty$ the function $W$ may change the sign. In our case $W$ is cubic in the square root of $m$ and may have at most three zeros which divide $\mathbb{R}$ into intervals where $W$ is either positive or negative. If $W>0$ or $W<0$ then we must choose the + or - sign in equations (44), (46), (47), and (50). This defines the type of the solution (static or homogeneous) on each interval. The corresponding solution yields the conformal building block for the construction of the CarterPenrose diagram for a maximally extended solution. The modulus sign in the left-hand side of equation (47) is crucial. It means that for a given range of $m$ there are two solutions: $m(\zeta)$ and $m(-\zeta)$. That is, all solutions in the conformal gauge are encountered in pairs related to each other by a reflection $\tau \rightarrow-\tau$ or $\sigma \rightarrow-\sigma$. The zeros of $W$ define horizons separating the blocks. The gluing procedure is unique, and for given constants in $W$ one obtains a unique universal covering space-time. The details of this construction are exactly the same as for $2 d$ gravity with torsion [11] and are summarized in section 4.2.

An integration constant in the equation (47) corresponds to a shift of the coordinates $\tau$ or $\sigma$ and will be always set to zero. For the scalar curvature of the surface $\mathbb{U}$ one finds (using (35), (46) and (47))

$$
R^{g}=\frac{2}{3} \Lambda+\frac{4 M}{|m|^{3 / 2}}
$$

It does not depend on $K$ or on the form of $W$ in (48), (49), and is singular at $m=0$ if $M \neq 0$. The singular part of (51) gives the eigenvalue of the singular four-dimensional Weyl tensor [19].

$$
\frac{1}{48} \widehat{C}_{i j k l} \widehat{C}^{i j k l}=\left(-\frac{M}{|m|^{3 / 2}}\right)
$$

\subsection{Schwarzschild coordinates}

At this point we have reduced the whole set of the Einstein equations to the first order ordinary differential equation (47), the line element being given by eq. (50). In general, equation (47) cannot be integrated in terms of elementary functions but its solutions can easily be analysed qualitatively. In this way we shall classify all global solutions in the next sections. However, local solutions of the Einstein equations may be obtained in explicit form without solving (47). This is achieved by using Eddington-Finkelstein or Schwarzschild coordinates. 
The transformation to Schwarzschild coordinates is very simple. There are two cases: static and homogeneous solutions. For a static solution the function $m(\sigma)$ depends on a spacelike coordinate. Restricting ourselves to positive $m$, because of the curvature singularity at $m=0$, and setting $m=-r^{2}, r(\sigma)>0$, equation (47) becomes

$$
\left|\frac{d r}{d \sigma}\right|=N(r)
$$

where the right-hand side

$$
N(r)=K-\frac{2 M}{r}-\frac{\Lambda r^{2}}{3}
$$

must be positive. This requirement defines the range of $r$ for a given $\Lambda, K$, and $M$. The corresponding line element reads

$$
d s^{2}=\left|\frac{d r}{d \sigma}\right|\left(d \tau^{2}-d \sigma^{2}\right)-r^{2} d \Omega^{2} .
$$

Substituting $(\tau, \sigma)$ by $(\tau, r)$ this becomes

$$
d s^{2}=N(r) d \tau^{2}-\frac{d r^{2}}{N(r)}-r^{2} d \Omega^{2}, \quad N>0,
$$

where $N$ is given by equation (54). In the case of spherical symmetry, that is $K=1$, it was first found by Kottler [20 and is well known to be of type $D$. For $\Lambda=0$ it reduces to the usual form of the Schwarzschild solution, $M$ being interpreted as the mass. The form of the angular part of the line element allows one to interpret $r$ as the radius in the spherical coordinate system. This works only for a static branch of the solution where $N>0$, i.e. outside of the horizon, $r>2 M$. The timelike coordinate in the solution (55) is not restricted at all, $\tau \in(-\infty, \infty)$.

Inside the horizon the solution is homogeneous, $m=m(\tau)$ and we set $m=$ $-t^{2}, t(\tau)>0$. Then equation (47) reduces to

$$
\left|\frac{d t}{d \tau}\right|=-N(t), \quad N<0,
$$

where

$$
N(t)=K-\frac{2 M}{t}-\frac{\Lambda t^{2}}{3} .
$$

The metric in Schwarzschild coordinates $(t, \sigma)$ reads

$$
d s^{2}=-\frac{d t^{2}}{N(t)}+N(t) d \sigma^{2}-t^{2} d \Omega^{2}, \quad N<0 .
$$


The range of the timelike coordinate $t$ is defined by the inequality $N<0$ and the singularity at $t=0$, i.e. $0<t<2 M$.

In Schwarzschild coordinates it is not necessary to solve equation (53) or (56). They simply define the transformation to conformally flat coordinates on $\mathbb{U}$. But also in conformal coordinates we do not need the explicit solution to equation (53). To analyse the behaviour of extremals and to construct global solutions only the behaviour of $W(m)$ near its zeros and its asymptotic behaviour as $m \rightarrow \infty$ and $m \rightarrow 0$ are needed.

The analysis of this section may be considered as a kind of uniqueness theorem stating that among the warped metrics the Schwarzschild solution is the only static black hole configurations in vacuum with zero cosmological constant [21] (for a review, see [22]). Indeed, spherical symmetry was not assumed for the metric (11). As a consequence of the Einstein equations (2) we have found that there is a solution with $R^{h}=-2 K=$ const. For $K=1$ the surface $\mathbb{V}$ must be a sphere $\mathbb{S}^{2}$. Therefore the solution must be spherically symmetric, and one immediately obtains the Schwarzschild solution.

\subsection{Global structure}

Solutions of the Einstein equations for $k=1$ are parametrized by three parameters. The cosmological constant $\Lambda$ is arbitrary. The scalar curvature $K$ of the surface $\mathbb{V}$ essentially takes three values $K=-1,0,1$. The third parameter $M$ is a constant of motion and may be arbitrary. For the Schwarzschild solution it is interpreted as the mass, and a physically acceptable range is $M>0$. Nevertheless, at least for comparison we classify solutions for all values of $M$. The maximally extended constant curvature surface $\mathbb{V}$ is a sphere, a plane (resp. a cylinder or a torus) or a Lobachevsky plane (resp. a higher genus Riemannian surface) for $K=1,0,-1$, respectively. Therefore, the classification of the global solutions reduces to the classification of the maximally extended surfaces $\mathbb{U}$ with Lorentzian metric. These surfaces are represented globally by Carter-Penrose diagrams.

A Carter-Penrose diagram is a diffeomorphic image of a maximally extended surface on a plane, such that the two sets of null extremals are represented by two sets of perpendicular lines $\left( \pm 45^{\circ}\right)$ as for the Minkowskian plane. It has global time and space orientation. The diagram consists of three types of conformal blocks shown in Fig. 1. Each block corresponds to a conformally flat metric

$$
d s^{2}=N(q)\left(d \tau^{2}-d \sigma^{2}\right)
$$

where $q$ is related to $\zeta=\sigma$ or $\tau$ through (53) resp. (56), i.e.

$$
\left|\frac{d q}{d \zeta}\right|= \pm N(q) .
$$




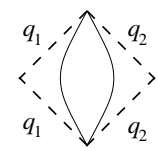

(a)

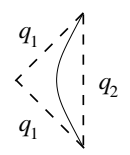

(b)

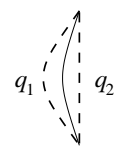

(c)

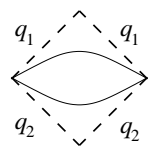

(d)

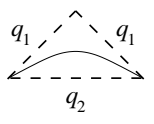

(e)

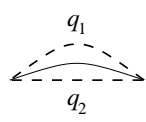

$(f)$

Figure 1: Conformal blocks for static $(a, b, c)$ and homogeneous $(d, e, f)$ solutions. Thin full lines indicate the direction of the Killing vector fields. The value of $q$ is constant along trajectories and varies smoothly from the left to the right boundary for static solutions, being constant at the boundaries. It varies in a similar way but from the lower to the upper boundary for the homogeneous solutions. Each conformal block has its symmetric partner with left and right resp. lower and upper boundaries interchanged.

Due to (51) the parameter $q$ is related to the scalar curvature of $\mathbb{U}$ by a simple algebraic relation. Thus the metric (59) is fully defined by the scalar curvature $R^{g}$. If $N>0$ or $N<0$ then the conformal block is called static or homogeneous, respectively. We say that a static block has left and right boundary whereas a homogeneous one has lower and upper boundary. The parameter $q$ varies within a conformal block, being constant along the integral curves of Killing vector fields and the boundaries. To each conformal block corresponds a time or space reversed symmetric partner. The range of the coordinates $\sigma$ and $\tau$ is determined by the zeros of the conformal factor, $N\left(q_{k}\right)=0$, called horizons, and the two boundary values $q_{0}$ and $q_{\infty}$ of the maximally extended surface corresponding either to a singularity or to a complete boundary where the integral (metric distance)

$$
\int d s=\int^{q_{\infty}} \frac{d q}{\sqrt{|N(q)|}}
$$

diverges. In our case $q_{\infty}=\infty$, but in general the infinite boundary with respect to the metric (59) may correspond to a finite value of $q$. The singularity may lie either at a finite or an infinite distance depending on the integral (61). There are three types of conformal blocks: diamond, triangle, and "eye" shown in Fig. 1. If the coordinates $\sigma, \tau$ cover the whole plane, then the solution (59) is mapped onto a finite diamond, Fig. $1(a)$ and $(d)$, by the conformal transformation. If the coordinates cover a half plane, i.e. the integral

$$
\int d \zeta=\int^{q_{0}, q_{k} \text { or } q_{\infty}} \frac{d q}{N(q)}
$$

converges at one end (boundary value or horizon) of the respective $q$-interval, then the solution is represented by a triangular conformal block, Fig.1 (b) and 
(e). If the function $N$ does not have any zero and the integral (61) converges both at $q_{0}$ and $q_{\infty}$, then the maximally extended surface is given by an "eye" Carter-Penrose diagram shown in Fig.1 $(c)$ and $(f)$. Now we give the rules for construction of a maximally extended solution for a given metric of the form (59) [11]:

- Consider a range of the parameter $q$ between two boundary values $q_{0}$ and $q_{\infty}$, where the curvature is singular or the integral (61) diverges. Call the corresponding boundaries singular and infinite, respectively.

- Find roots of the equation $N\left(q_{k}\right)=0, k=1, \ldots, n$ between $q_{0}$ and $q_{\infty}$ and call them horizons, the degree of the zero $q_{k}$ being the degree of the corresponding horizon.

- For each of the intervals $\left(q_{0}, q_{1}\right), \ldots,\left(q_{n}, q_{\infty}\right)$ draw a pair of static or homogeneous conformal blocks for $N>0$ and $N<0$, respectively.

- If there are horizons, then glue conformal blocks along horizons preserving the smoothness of $N$. That is, the boundaries of conformal blocks must be glued together, corresponding only to the adjacent intervals having $q_{k}$ as a boundary point.

- For a given $N$ the Carter-Penrose diagram constructed by gluing all adjacent but different conformal blocks constitutes a fundamental region. If $N \geq 0$ or $N \leq 0$ everywhere between $q_{0}$ and $q_{\infty}$ (that is all possible horizons are of even degree), then there are two disconnected fundamental regions related by space or time reflection.

- If the boundary of the fundamental region consists of the boundaries of the conformal blocks corresponding to $q_{0}$ or $q_{\infty}$ then the fundamental region represents the unique smooth maximally extended surface.

- If the boundary of the fundamental region includes horizons then the fundamental region may be continued periodically in time or space or both directions simultaneously, or the opposite sides may be identified.

The final step is to determine the completeness or incompleteness of the singular boundary by considering the integral (61) at $q_{0}$. There are curious situations. For example, in ordinary dilaton gravity the singularity is complete for null extremals but incomplete for time-like ones [5]. The inner points where horizons cross may be at a finite or infinite distance depending on whether the integral (61) converges or diverges at $q_{k}$, i.e. they are at an infinite distance iff $q_{k}$ is a zero of degree $\geq 2$. Such infinite points inside the diagram do occur in $2 d$ gravity with torsion [11]. The present case is simpler, and all points inside a diagram are finite.

Near a horizon the function $N$ behaves like $\left(q-q_{k}\right)^{a}$. For $a \geq 1$ the integral (62) diverges. Therefore conformal blocks between such horizons are of diamond 
type. If horizons are absent then the metric (59) covers the whole maximally extended space-time and is represented by one conformal block.

The described procedure is quite general, unique and yields a globally smooth maximally extended solution. The advantage of this constructive approach is that the global structure of the space-time is defined without explicit construction of a global coordinate system. The starting point is the local form of the metric admitting one Killing vector field. The global $\mathcal{C}^{\infty}$ smoothness can be proved by explicit construction of the Eddington-Finkelstein and Kruskal-Szekeres coordinates covering the fundamental region. In our present paper we work with the conformal approach, the main reason being that we have to treat Euclidean metrics as well, where the Eddington-Finkelstein coordinates do not exist.

Thus the classification of global solutions to the Einstein equations reduces to the analysis of the zeros of $N$ for different values of $\Lambda, K$, and $M$. The function $q N(q)$ is cubic in $q$ and may have up to three zeros. One of the zeros is necessarily negative and should be dropped. Thus, there occur at most two horizons. Below we shall write down explicitly the local solutions of Einstein's equations in Schwarzschild coordinates and in the conformal gauge where equation (60) can be solved using elementary functions. For $\Lambda=0, K=0$, and $M=0$ we have $m=$ const, and the space-time is Minkowskian, as found in Section 3. Hence we assume that at least one parameter differs from zero. We classify the solutions according to the scalar curvature of the $\mathbb{V}$ surface.

\subsection{Spherically symmetric solutions, $K=1$}

For $K=1$ the surface $\mathbb{V}=\mathbb{S}^{2}$ is a sphere and all solutions are spherically symmetric. For the unit sphere the metric (30) may be rewritten in spherical coordinates

$$
d \Omega^{2}=d \theta^{2}+\sin ^{2} \theta d \varphi^{2}
$$

The solutions are parametrized by a cosmological constant $\Lambda$ and a mass $M$. The line element in Schwarzschild coordinates has the usual form

$$
d s^{2}=N(q) d \zeta^{2}-\frac{d q^{2}}{N(q)}-q^{2}\left(d \theta^{2}+\sin ^{2} \theta d \varphi^{2}\right),
$$

where

$$
N(q)=1-\frac{2 M}{q}-\frac{\Lambda q^{2}}{3} .
$$

Depending on the sign of $N$, the coordinates $q$ and $\zeta$ may be space- or timelike

$$
\begin{array}{lll}
N(q)>0, & q=r, & \zeta=\tau, \\
N(q)<0, & q=t, & \zeta=\sigma .
\end{array}
$$


The parameter $q$ must be positive. Despite the similar local form of the solution for different values of $\Lambda$ and $M$, the global structure varies qualitatively. It depends on the number and types of the roots of the equation $N(q)=0$ or

$$
\frac{\Lambda}{3} q^{3}-q+2 M=0
$$

For nonzero cosmological constant (67) has up to three zeros. Elementary analysis shows that one root is always negative and we have at most two positive roots and, consequently, two horizons. For positive cosmological constant, $\Lambda>0$, we have the following possibilities: For $3 M>\frac{1}{\sqrt{\Lambda}}$ equation (67) has no positive root. If $3 M=\frac{1}{\sqrt{\Lambda}}$, then there is one double positive root. In the interval $0<3 M<\frac{1}{\sqrt{\Lambda}}$ there are two positive roots. For $M \leq 0$ we have one positive root. For negative cosmological constant, $\Lambda<0$, there is one positive root for $M>0$ and no positive root for $M<0$.

\subsubsection{Minkowskian space-time, $\Lambda=0, M=0$}

The simplest spherically symmetric solution is obtained for $\Lambda=0$ and $M=$ 0 (cf. Fig. $2(a)$ ). Then $N=1$, and the line element in the Schwarzschild coordinates becomes

$$
d s^{2}=d \tau^{2}-d r^{2}-r^{2}\left(d \theta^{2}+\sin ^{2} \theta d \varphi^{2}\right), \quad 0<r<\infty .
$$

The point $r=0$ is a coordinate singularity. Going to four-dimensional Cartesian coordinates and adding the time developed origin, $r=0$, we get the fourdimensional Minkowskian space-time. Here the spacelike coordinate $r$ is naturally identified with the radius of the spherical coordinate system.

\subsubsection{Schwarzschild black hole solution, $\Lambda=0, M>0$}

The Schwarzschild solution corresponds to zero cosmological constant, $\Lambda=0$, and positive mass, $M>0$. Its Carter-Penrose diagram is well-known. Nevertheless, it represents a suitable non-trivial illustration of our general approach. In this case the right-hand side of the equation (53)

$$
\left|\frac{d r}{d \sigma}\right|=1-\frac{2 M}{r},
$$

has one zero at $r_{1}=2 M$, so there is one horizon. For definiteness we assume here that $d r / d \sigma>0$ and then add solutions with $d r / d \sigma<0$ by reflection $\sigma \rightarrow-\sigma$. In the region $N>0$ i.e. $r>2 M$ the solution is static. For $r=r(\sigma)$ equation (69) yields

$$
r+2 M \ln (r-2 M)=\sigma, \quad-\infty<\sigma<\infty
$$




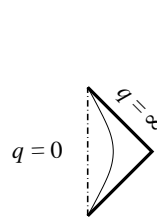

(a)

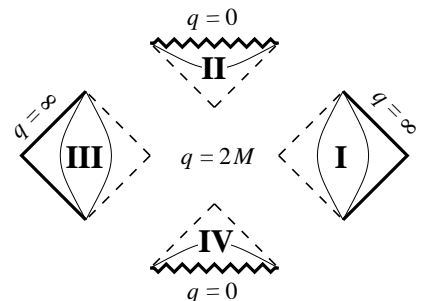

$\left(b_{1}\right)$

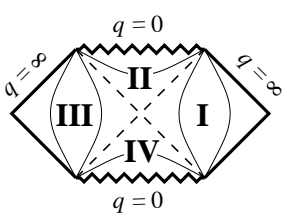

$\left(b_{2}\right)$

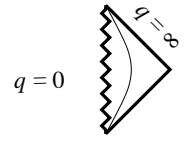

(c)

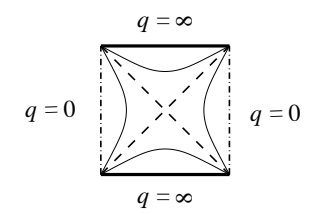

$(d)$

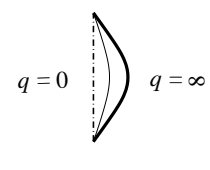

(e)

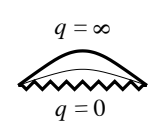

$(f)$

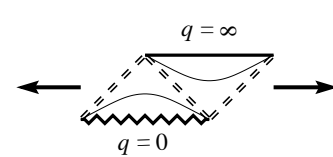

$(g)$

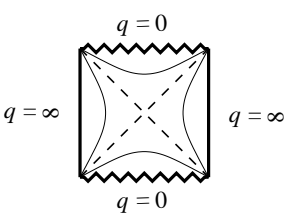

(j)

Figure 2: Spherically symmetric solutions, $K=1$. In our notations the zigzag lines denote the singular boundary lying at a finite distance, solid lines denote a regular infinite boundary, thin lines are the Killing trajectories, dashed lines denote horizons. Solid arrows indicate a possible periodic continuation of the fundamental regions.

revealing $\sigma$ as the "tortoise coordinate". The corresponding line element in the conformal gauge has the form

$$
d s^{2}=\left(1-\frac{2 M}{r}\right)\left(d \tau^{2}-d \sigma^{2}\right)-r^{2} d \Omega^{2}
$$

where $r(\sigma)$ is given implicitly by (70), or in Schwarzschild coordinates

$$
d s^{2}=\left(1-\frac{2 M}{r}\right) d \tau^{2}-\frac{d r^{2}}{1-\frac{2 M}{r}}-r^{2} d \Omega^{2}, \quad 2 M<r<\infty
$$

The corresponding conformal blocks of a Carter-Penrose diagram are the diamonds I, III shown in Fig. $2\left(b_{1}\right)$. In the region $N<0$ or $0<t<2 M$ the solution depends on the time $t=t(\tau)$, and we have to change the sign of the 
right-hand side of equation (69). This implicitly defines $t(\tau)$ :

$$
t+2 M \ln (2 M-t)=-\tau, \quad-2 M \ln (2 M)<\tau<\infty
$$

In conformal gauge resp. in Schwarzschild coordinates the line element reads

$$
\begin{aligned}
& d s^{2}=-\left(1-\frac{2 M}{t}\right)\left(d \tau^{2}-d \sigma^{2}\right)-t^{2} d \Omega^{2} \\
& d s^{2}=-\frac{d t^{2}}{1-\frac{2 M}{t}}+\left(1-\frac{2 M}{t}\right) d \sigma^{2}-t^{2} d \Omega^{2}, \quad 0<t<2 M
\end{aligned}
$$

The corresponding conformal blocks are now a triangle and its reflection as shown in Fig. $2\left(b_{1}\right)$ II, IV. The corresponding Carter-Penrose diagram for the global solution is unique and shown in Fig. $2\left(b_{2}\right)$.

\subsubsection{Naked singularity, $\Lambda=0, M<0$}

For negative mass the right-hand side of equation (69) is positive for $r>0$ and the solution depends on a spacelike coordinate $\sigma$. The line element has the same form (71) as for the Schwarzschild solution. The only difference is the range of $\sigma \in(2 M \ln |2 M|, \infty)$. Thus there is only one triangular conformal block shown in Fig. $2(c)$ and its space reflection. Each of them represents the maximally extended surface $\mathbb{U}$.

\subsubsection{De Sitter solution, $\Lambda>0, M=0$}

The de Sitter solution corresponds to a positive cosmological constant and zero mass. This complete constant curvature manifold may also be represented as the "unit" hyperboloid embedded in 5-dimensional Minkowski space with the induced metric. Consequently, its symmetry group equals $O(1,4)$, and it has the maximal number of 10 Killing fields. The function (65) has one zero so there is one horizon. The static and homogeneous solutions in Schwarzschild coordinates become

$$
\begin{aligned}
d s^{2}= & \left(1-\frac{\Lambda}{3} r^{2}\right) d \tau^{2}-\frac{d r^{2}}{1-\frac{\Lambda}{3} r^{2}}-r^{2} d \Omega^{2}, \\
& 0<r<\sqrt{\frac{3}{\Lambda}}, \quad-\infty<\tau<\infty \\
d s^{2}= & -\frac{d t^{2}}{1-\frac{\Lambda}{3} t^{2}}+\left(1-\frac{\Lambda}{3} t^{2}\right) d \sigma^{2}-t^{2} d \Omega^{2} \\
& \sqrt{\frac{3}{\Lambda}}<t<\infty, \quad-\infty<\sigma<\infty
\end{aligned}
$$


Since 60) can be integrated in that case, the line elements may be written explicitly in conformal gauge, too,

$$
\begin{aligned}
d s^{2} & =\frac{1}{\cosh ^{2}\left(\sqrt{\frac{\Lambda}{3}} \sigma\right)}\left(d \tau^{2}-d \sigma^{2}\right)-\frac{3}{\Lambda} \tanh ^{2}\left(\sqrt{\frac{\Lambda}{3}} \sigma\right) d \Omega^{2} \\
d s^{2} & =\frac{1}{\sinh ^{2}\left(\sqrt{\frac{\Lambda}{3}} \tau\right)}\left(d \tau^{2}-d \sigma^{2}\right)-\frac{3}{\Lambda} \operatorname{coth}^{2}\left(\sqrt{\frac{\Lambda}{3}} \tau\right) d \Omega^{2}
\end{aligned}
$$

for static and homogeneous solutions, respectively. The range $r \in(0, \sqrt{3 / \Lambda})$ is transformed to $\sigma \in(0, \infty)$ and $t \in(\sqrt{3 / \Lambda}, \infty)$ to $\tau \in(0, \infty)$. Both line elements (78) and (79) describe triangular conformal blocks, and the CarterPenrose diagram is shown in Fig. $2(d)$. Adding the line $r=0$ (i.e. the timeevolved coordinate origin) to the warped product $\mathbb{U} \times \mathbb{S}^{2}$, the de Sitter solution is obtained.

\subsubsection{Anti de Sitter solution, $\Lambda<0, M=0$}

Changing the sign of the cosmological constant in the Einstein equations results in a qualitatively different solution. For zero mass we have the anti de Sitter solution which may also be represented as a "unit" hyperboloid, but this time embedded in a flat five-dimensional space of signature $(++---)$. The corresponding symmetry group is $O(2,3)$. The function (65) has no zeros and is always positive. Therefore the solution is static and has no horizon. In Schwarzschild coordinates the line element has the same form as for the de Sitter solution (76) but due to the minus sign of $\Lambda$ the range of $r$ is now $(0, \infty)$. In the conformal gauge we obtain

$$
d s^{2}=\frac{1}{\cos ^{2}\left(\sqrt{\frac{|\Lambda|}{3} \sigma}\right)}\left(d \tau^{2}-d \sigma^{2}\right)-\frac{3}{|\Lambda|} \tan ^{2}\left(\sqrt{\frac{|\Lambda|}{3}} \sigma\right) d \Omega^{2}
$$

Here $\sigma$ runs through the finite interval $\sigma \in\left(0, \frac{\pi}{2} \sqrt{\frac{3}{|\Lambda|}}\right)$. In this case we have an "eye" diagram shown in Fig. $2(e)$, but we could equally well draw it as an infinite ribbon with vertical boundary lines. This manifold is incomplete at $r=0$. To obtain the entire anti de Sitter solution we have to add this line (time-developed point) to the manifold.

\subsubsection{Homogeneous space singularity, $\Lambda>0,3 M>\frac{1}{\sqrt{\Lambda}}$}

For these values of the constants the function $N$ has no zero for $q>0$ and is always negative. The solution is homogeneous without horizons. The surface $\mathbb{U}$ in that case is represented by an "eye" Penrose diagram shown in Fig. $2(f)$ 
and its time reversal. At a finite past we observe a true singularity both in four-dimensional curvature and the curvature of the surface $\mathbb{U}$, and the manifold cannot be extended through it. A cosmological interpretation of this diagram implies a Universe born at a finite past lasting forever. The surface $\mathbb{U}$ is asymptotically de Sitter as $t \rightarrow \infty$. The same is also true for the three cases described below.

\subsubsection{One double horizon, $\Lambda>0,3 M=\frac{1}{\sqrt{\Lambda}}$}

In that case the function $N$ has one double zero at $t=1 / \sqrt{\Lambda}$ corresponding to a horizon, and the function $N$ is everywhere negative for $q>0$. The Carter-Penrose diagram is the infinite ribbon shown in Fig. $2(g)$ and its time reversal.

\subsubsection{Two horizons, $\Lambda>0,0<3 M<\frac{1}{\sqrt{\Lambda}}$}

The function $N$ has two zeros (two horizons). The Carter-Penrose diagram consists of two homogeneous and one static conformal block. The corresponding universal covering space is an infinite ribbon shown in Fig. $2(h)$.

\subsubsection{Two static singularities $\Lambda>0, M<0$}

For negative mass the function $N$ has one zero. The maximally extended solution is shown in Fig. $2(i)$. We have two static singular regions I,III separated by horizons. From I only the right-hand singularity can be reached by a causal path and the same applies to III and the left-hand singularity. Region IV has access to both (naked) singularities, whereas from II no singularity can be reached.

\subsubsection{Anti de Sitter black hole $\Lambda<0, M>0$}

In that case the function $N$ has one zero, that is, one horizon. The Carter-Penrose diagram is shown in Fig. $2(j)$, and is similar to the one for the Schwarzschild black hole except that the complete left and right boundaries are now timelike. The singularities at finite past and finite future are similar to those of the Schwarzschild solution. The space-time is not asymptotically flat but becomes anti de Sitter as $r \rightarrow \infty$.

\subsubsection{1 $\Lambda<0, M<0$}

This is a naked singularity without horizon. Its Carter-Penrose diagram equals the one in Fig. $2(f)$ but turned by $\pi / 2$ as the solution is static. The space at $r \rightarrow \infty$ is asymptotically anti de Sitter again. 


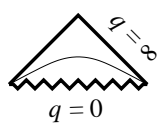

(a)

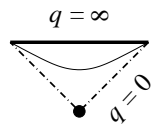

(b)

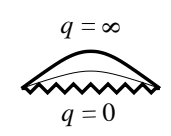

(c)

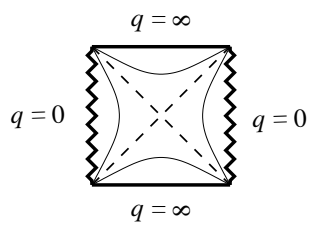

(d)

Figure 3: Planar solutions, $K=0$.

\subsection{Planar solutions, $K=0$}

In the case $K=0$ the line element (30) is

$$
d \Omega_{P}^{2}=d y^{2}+d z^{2}
$$

and describes the Euclidean plane with the symmetry group $I O(2)$ or its compactified versions: a cylinder or a torus. In the planar case the line element in Schwarzschild coordinates is

$$
d s^{2}=N(q) d \zeta^{2}-\frac{d q^{2}}{N(q)}-q^{2}\left(d y^{2}+d z^{2}\right)
$$

where

$$
N(q)=-\frac{2 M}{q}-\frac{\Lambda q^{2}}{3} .
$$

Coordinates $q$ and $\zeta$ are the same as in equation (66). The global solutions depend on $M$ and $\Lambda$.

The physical interpretation of these solutions is quite interesting. For instance, the whole three-dimensional space of the corresponding solutions may be a product of a torus $\mathbb{T}^{2}$ with a line $\mathbb{R}$ (for the choice $\mathbb{V}=\mathbb{T}^{2}$ ). This space contains non-contractible loops (nontrivial fundamental group) and may be interpreted as a wormhole. Note that the horizon in that case will be a torus too.

\subsubsection{Homogeneous and naked singularities $\Lambda=0, M \neq 0$}

For zero cosmological constant and positive mass $M>0$ we have a homogeneous spacelike singularity. In that case $N$ is everywhere negative, and the solution does not have a horizon. In the Schwarzschild and conformal coordinates resp. the line elements are

$$
\begin{aligned}
& d s^{2}=\frac{t}{2 M} d t^{2}-\frac{2 M}{t} d \sigma^{2}-t^{2} d \Omega_{P}^{2}, \quad 0<t<\infty, \\
& d s^{2}=\sqrt{\frac{M}{|\tau|}}\left(d \tau^{2}-d \sigma^{2}\right)-4 M|\tau| d \Omega_{P}^{2}, \quad \tau<0 \quad \text { or } \quad \tau>0 .
\end{aligned}
$$


The corresponding Carter-Penrose diagram is shown in Fig. 3 (a). As usual, there is also another time reversed solution.

For negative mass, $M<0$, the solution has a similar form but is stationary. Then the Carter-Penrose diagram should be turned by $\pi / 2$ and describes a naked singularity.

\subsection{2 (Anti) de Sitter solution, $\Lambda \neq 0, M=0$}

For positive cosmological constant $(\Lambda>0)$ and zero mass the function

$$
N=-\frac{\Lambda}{3} t^{2}
$$

is everywhere negative and has no zeros. The corresponding solution is homogeneous without horizon. In the Schwarzschild and conformal coordinates it reads

$$
\begin{aligned}
d s^{2} & =\frac{3}{\Lambda t^{2}} d t^{2}-\frac{\Lambda t^{2}}{3} d \sigma^{2}-t^{2} d \Omega_{P}^{2} \\
d s^{2} & =\frac{3}{\Lambda \tau^{2}}\left(d \tau^{2}-d \sigma^{2}\right)-\frac{9}{\Lambda^{2} \tau^{2}} d \Omega_{P}^{2}
\end{aligned}
$$

The last equation shows that this solution is conformally flat Along the boundary lines $q=0$ (i.e., $t=0$ ) of the Carter-Penrose diagram (Fig. $3(b)$ ) our $4 d$-metric becomes degenerate. One can verify that metrics (86) and (87) describe spacetime of constant curvature. This means that these metrics represent (part of) the de Sitter solution.

The case of a negative cosmological constant, $\Lambda<0$, is similar but one has to replace the time dependence of metric components in eqs. (86) and (87) by a space dependence and turn the Carter-Penrose diagram by $\pi / 2$. This is one of the forms of anti de Sitter space-time.

\subsection{3 $\Lambda \neq 0, M \neq 0$}

If $\Lambda>0$ and $M>0$ the function

$$
N=-\frac{\Lambda t^{3}+6 M}{3 t}<0
$$

is negative without zeros. In Schwarzschild resp. conformal coordinates the line elements are

$$
\begin{aligned}
d s^{2} & =\frac{3 t}{\Lambda t^{3}+6 M} d t^{2}-\frac{\Lambda t^{3}+6 M}{3 t}-t^{2} d \Omega_{P}^{2}, \quad t \in(0, \infty) . \\
d s^{2} & =\left|\frac{d t}{d \tau}\right|\left(d \tau^{2}-d \sigma^{2}\right)-t^{2} d \Omega_{P}^{2}, \quad-\frac{\pi}{12 \sqrt{3} M}<\tau<\frac{\pi}{4 \sqrt{3} M} .
\end{aligned}
$$


Here the function $t=t(\tau)$ is defined implicitly by the relation

$$
\frac{\Lambda}{3} \tau=\frac{1}{6 a} \ln \frac{a^{2}-a t+t^{2}}{(a+t)^{2}}+\frac{1}{a \sqrt{3}} \arctan \left(\frac{2 t-a}{a \sqrt{3}}\right), \quad a=6 M / \Lambda .
$$

Correspondingly, the inextendible solution is represented by an "eye" diagram as shown in Fig. $3(c)$, and its time reversal. It describes a homogeneous space singularity at $t=0$ and is asymptotically de Sitter as $t \rightarrow \infty$.

If $\Lambda>0, M<0$, the function (88) has one simple zero corresponding to a horizon. There is one static and one homogeneous conformal block (and their reflections) forming together the Carter-Penrose diagram shown in Fig. $3(d)$. It coincides with the diagram for the spherically symmetric case $K=1, \Lambda>0$, and $M<0$ (Fig. $2(i)$ ). The line element for the homogeneous block has the same form as (89) but due to (91) the ranges of $t$ and $\tau$ differ. For the static block the line element is given by (55) with the appropriate ranges for $r$ and $\sigma$ related by the same equation as $t$ and $\tau$.

The cases $\Lambda<0, M<0$ and $\Lambda<0, M>0$ are obtained from the cases $\Lambda>0$, $M>0$ and $\Lambda>0, M<0$ by exchanging timelike and spacelike coordinates. The last case corresponds to a torus black hole.

\subsection{Lobachevsky plane solutions, $K=-1$}

For $K=-1$ the surface $\mathbb{V}$ is a Lobachevsky plane or, in a compactified version, a higher genus Riemannian surface. Note that the Lorentz transformation group $O(1,2)$ acts on a spacelike surface in that case. The line element (30) in angular coordinates is

$$
d \Omega_{L}^{2}=d \theta^{2}+\sinh ^{2} \theta d \varphi^{2}
$$

The corresponding vacuum solution to the Einstein equation has the form

$$
d s^{2}=N(q) d \zeta^{2}-\frac{d q^{2}}{N(q)}-q^{2}\left(d \theta^{2}+\sinh ^{2} \theta d \varphi^{2}\right),
$$

where

$$
N(q)=-1-\frac{2 M}{q}-\frac{\Lambda q^{2}}{3}
$$

The function $W$ as given by (49) changes its overall sign under the reflection $K \rightarrow-K, \Lambda \rightarrow-\Lambda$, and $M \rightarrow-M$. Thus the classification of solutions for $K=-1$ is the same as for the spherically symmetric case if one interchanges space and time coordinates on the $\mathbb{U}$ surface, $\tau \leftrightarrow \sigma$, and changes the sign of cosmological constant and mass. For example, the analog of the Schwarzschild 
solution is obtained for $\Lambda=0$ and $M<0$. For homogeneous and static conformal blocks the line element is

$$
\begin{gathered}
d s^{2}=\frac{d t^{2}}{1+\frac{2 M}{t}}-\left(1+\frac{2 M}{t}\right) d \sigma^{2}-t^{2} d \Omega^{2}, \quad-2 M<t<\infty, \\
d s^{2}=\left(1+\frac{2 M}{r}\right) d \tau^{2}-\frac{d r^{2}}{1+\frac{2 M}{r}}-r^{2} d \Omega^{2}, \quad 0<r<-2 M .
\end{gathered}
$$

The corresponding Carter-Penrose diagram must be turned by the angle $\pi / 2$. There are left and right static singularities at $r=0$. The properties of this global solution are similar to the properties of two static singularities in the spherically symmetric case, but with a different "asymptotic" behaviour: the space-time is now asymptotically flat at infinite past and infinite future (both limits correspond to $t \rightarrow \infty)$.

It is not easy to imagine a space (a time slice of the four-dimensional spacetime defined by the equation $t=$ const) for $K=-1$. In an uncompactified version it is the product of an interval (finite or infinite depending on the values of $\Lambda$ and $M)$ and the Lobachevsky plane. In a compactified version when $\mathbb{V}$ is a higher genus Riemann surface one may interpret this class of solutions as describing a set of wormholes, the number of wormholes being exactly the number of handles of the corresponding Riemann surface. The same topology will be inherited by a horizon. In the same way one may analyse the other solutions for $K=-1$, but we shall not repeat this analysis here.

\section{Hyperbolic solutions (case $C$ ).}

Whereas solutions of the last section are known, the global properties of the solutions to be discussed now seem to be new. Their topological structure is $\mathbb{M}=\mathbb{H}^{2} \times \mathbb{V}$, where $\mathbb{H}^{2}$ is a one-sheet hyperboloid embedded in three-dimensional Minkowski space (or its universal covering, resp.) and $\mathbb{V}$ is a Euclidean surface to be specified below. This class of vacuum solutions to the Einstein equations yields solutions with curvature or conical singularities located along space-like strings or domain walls of curvature singularity evolving in time. All singularities are naked ones.

Case $C$ is very similar to the spatially symmetric solutions (case $B$ ) but has important new features. First, we cannot restrict ourselves to positive definite metrics $h_{\mu \nu}$ on $\mathbb{V}$ because one of the Einstein equations (22) is not invariant under the transformation $h_{\mu \nu} \rightarrow-h_{\mu \nu}$ for a fixed $m$. Note that in the case $k=$ const the transformation $g_{\alpha \beta} \rightarrow-g_{\alpha \beta}$ may be always compensated by exchanging space and time coordinates on $\mathbb{U}, \tau \leftrightarrow \sigma$ leaving equation (21) invariant. This is impossible for the Euclidean signature metric on $\mathbb{V}$. Therefore we fix $m=1$ to have the 
same signs in the equation as in the case $k=1$, but allow the metric $h_{\mu \nu}$ to be both positive or negative definite.

To obtain solutions of equations (21)-(24) one may follow the same steps as before replacing everywhere $m$ by $k$ and $g_{\alpha \beta}$ by $h_{\mu \nu}$. Therefore we merely sketch the whole procedure stressing only particularly important points resulting from the Euclidean signature. Now the conformal gauge is

$$
h_{\mu \nu} d y^{\mu} d y^{\nu}=2 h d z d \bar{z}=2 h\left(d \rho^{2}+d \sigma^{2}\right)
$$

where $h(z, \bar{z})$ is a function of the complex coordinates on $\mathbb{V}$,

$$
z=\rho+i \sigma, \quad \bar{z}=\rho-i \sigma,
$$

where $\rho=y^{2}$, and $\sigma=y^{3}$. In the total line element

$$
d s^{2}=k d \Phi^{2}+2 h d z d \bar{z}
$$

$d \Phi^{2}$ is a constant curvature metric on $\mathbb{U}$ given, for example, by (29). For the two unknown functions $k$ and $h$ we have, instead of equations (41)-(43),

$$
\begin{aligned}
\partial_{z} \partial_{z} k-\frac{\partial_{z} k \partial_{z} k}{2 k}-\frac{\partial_{z} h \partial_{z} k}{h} & =0 \\
\partial_{\bar{z}} \partial_{\bar{z}} k-\frac{\partial_{\bar{z}} k \partial_{\bar{z}} k}{2 k}-\frac{\partial_{\bar{z}} h \partial_{\bar{z}} k}{h} & =0 \\
\frac{2 \partial_{z} \partial_{\bar{z}} k}{g}-2(k \Lambda+K) & =0 .
\end{aligned}
$$

The solution of (100) and (101) is $k=k(z \pm \bar{z}), h=h(z \pm \bar{z})$, and

$$
h= \pm \frac{\left|k^{\prime}\right|}{4 \sqrt{|k|}},
$$

where the upper and lower signs correspond to positive and negative definite metrics on $\mathbb{V}$, respectively. Thus, $k$ and $h$ depend on either $\rho$ or $i \sigma$. However, due to the rotational symmetry of (97) these two choices are equivalent and we assume for definitness that $k$ and $h$ are functions of $\rho$. Then the Laplacian is $4 \partial_{z} \partial_{\bar{z}} k=k^{\prime \prime}$ (no \pm sign!). Now instead of (46) we get

$$
k^{\prime \prime} \mp(k \Lambda+K) \frac{\left|k^{\prime}\right|}{\sqrt{|k|}}=0,
$$

with the same sign conventions as in (103). Using the result that the curvature has a true singularity at $k=0$ we restrict the range of $k$ to positive values. Then equation (104) may be integrated to give

$$
\left|\frac{d k}{d \rho}\right|=\mp 2 W(k),
$$


where

$$
W(k)=-\frac{1}{3} \Lambda k^{3 / 2}-K k^{1 / 2}-2 M, \quad k>0,
$$

$M$ being an arbitrary integration constant. Although it cannot be interpreted as a mass we use the same notation as before to simplify comparison. With the parametrization $k=r^{2}$ for positive $k$ a general hyperbolic solution to the Einstein equations is, finally,

$$
d s^{2}=r^{2} d \Phi^{2}-N(r)\left(d \rho^{2}+d \sigma^{2}\right)
$$

where

$$
N(r)=-K-\frac{2 M}{r}-\frac{1}{3} \Lambda r^{2},
$$

and the function $r=r(\rho)$ is defined by the equation

$$
\left|\frac{d r}{d \rho}\right|=\mp N(r) .
$$

The modulus sign in this equation means that in each case there are two solutions $r(\rho)$ and $r(-\rho)$. From equation (107) it is clear that for $N>0$ and $N<0$ the metric on $\mathbb{V}$ is negative and positive definite, respectively. Taking the function $r$ as a coordinate (107) can be written in Schwarzschild-like form

$$
d s^{2}=r^{2} d \Phi^{2}-\frac{d r^{2}}{N(r)}-N(r) d \sigma^{2}
$$

The resulting hyperbolic solution has three Killing vector fields generating the symmetry group $S O(1,2)$ of the one-sheet hyperboloid of constant curvature and one Killing vector field $\partial_{\sigma}$ for the $\mathbb{V}$ surface.

Calculations similar to the $k=1$ case yield explicit expression for the scalar curvature on $\mathbb{V}$,

$$
R^{h}=\frac{2}{3} \Lambda+\frac{4 M}{r^{3}}
$$

and for the invariant eigenvalue of the Weyl tensor we get the same expression as given in case $B$ by (52). This justifies the range of $k=r^{2} \in(0, \infty)$.

\subsection{Global structure}

The global structure of a hyperbolic solution (107) or (110) depends on the zeros of $N$ :

$$
N\left(r_{k}\right)=0 \text {. }
$$

The construction of maximally extended solutions requires a careful analysis of the extremals for a given metric. The local solutions of $2 d$ gravity with torsion 
have the same structure and therefore the analysis performed in [13] may be applied directly in the present case. To save space we only formulate the rules of how to construct maximally extended Euclidean solutions from the Lorentzian ones:

- Each of the Lorentz signature solution decomposes into disconnected solutions for the Euclidean metric along horizons. That is each conformal block by itself represents one maximally extended solution in the Euclidean case.

- Homogeneous, $N<0$, and static, $N>0$, conformal blocks represent surfaces of positive and negative definite metrics, respectively.

- Each of the horizons shrinks to a point in the Euclidean case and lies at a finite distance for a simple zero of $N$ and at infinite distance for a higher zero of $N$.

- The completeness or incompleteness of curvature singularities is the same as before.

To obtain a maximally extended Euclidean surface $\mathbb{V}$ one must identify the points $\sigma$ and $\sigma+L$ along the Killing direction [13] for a simple zero. The obtained surface is not a cylinder because its circumference shrinks to zero if one approaches a horizon, that is the zero of $N$. In general, one gets a conical singularity there with the deficit angle

$$
\triangle \omega=\frac{1}{2} L\left|N^{\prime}\left(r_{k}\right)\right|-2 \pi
$$

where $N^{\prime}$ denotes the derivative. Hence the necessary and sufficient condition for the absence of a conical singularity is

$$
L=\frac{2 \pi}{\left|\frac{M}{r_{k}^{2}}-\frac{1}{3} \Lambda r_{k}\right|} .
$$

If this equation holds then we get a smooth surface at this point and the whole space-time may be totally smooth (if there are no curvature or further conical singularities). For a double zero the points corresponding to this horizon lie at an infinite distance and one does not need to bother about conical singularities. Again the classification of global hyperbolic solutions depends on the number and type of zeros of $N$ defined by the constants $K, \Lambda$, and $M$.

\subsection{Hyperbolic solutions for $K=-1$}

Let us note that the cases $K=1$ and $K=-1$ may be obtained from each other by permutation of the first two coordinates. We choose $K=-1$ to retain the 
same expression for $N$ as for spherically symmetric solutions. The line element for the one sheet hyperboloid (29) in angular coordinates reads

$$
d \Phi^{2}=d \theta^{2}-\cosh ^{2} \theta d \varphi^{2}, \quad-\infty<\theta<\infty, \quad 0 \leq \varphi<2 \pi,
$$

so that the corresponding $4 d$ line element becomes

$$
d s^{2}=r^{2}\left(d \theta^{2}-\cosh ^{2} \theta d \varphi^{2}\right)-\frac{d r^{2}}{N(r)}-N(r) d \sigma^{2},
$$

where $N(r)$ is given by (65) again. It has precisely the same form as for the Kottler solution [20] but here it enters the Euclidean part of the metric. Note that now the surface $\mathbb{U}=\mathbb{H}^{2}$ or its universal covering space is completely smooth. The surface $\mathbb{V}$ may have a negative $(N>0)$ or positive $(N<0)$ definite metric. For negative definite metric the timelike coordinate is $\theta \in(-\infty, \infty)$, and the three-dimensional space is the product of a circle, $\varphi \in[0,2 \pi)$, and the surface $\mathbb{V}$ to be constructed below. If $\mathbb{U}$ is the universal covering space of the hyperboloid $\mathbb{H}^{2}$, then the space equals $\mathbb{R} \times \mathbb{V}$. The evolution of space in time lasts forever and if there are singularities, all of them are naked.

For positive definite metrics on $\mathbb{V}$ the timelike coordinate is $\varphi \in[0,2 \pi)$, and the space is a product of a line, $\theta \in(-\infty, \infty)$, with $\mathbb{V}$. The corresponding spacetime contains closed timelike curves (including extremals) unless $\mathbb{U}$ is chosen to be the universal covering of $\mathbb{H}^{2}$.

\subsubsection{Minkowskian space-time, $\Lambda=0, M=0$}

In this case the surface $\mathbb{V}$ is a half plane $r \in(0, \infty), \sigma \in(-\infty, \infty)$. It is incomplete at $r=0$ due to a coordinate singularity which was also the case for the spherical coordinates.

\subsection{2 $\Lambda=0, M>0$}

This is the counterpart of the Schwarzschild solution. There are two different and disconnected Euclidean surfaces shown in Fig. $4\left(a_{1}\right)$ and $\left(a_{2}\right)$ with negative and positive definite metric. There we have identified the points $\sigma$ and $\sigma+L$. The global solution corresponding to the surface $\left(a_{1}\right)$ has the signature $(+---)$ and is globally smooth or may contain a conical singularity at $r_{*}=2 M$. Topologically it is a plane and in the case of a conical singularity the whole space-time describes the infinitely long propagation of an infinite cosmic string. This solution has no further curvature singularity and if equation (111) is fullfiled the whole space-time is totally smooth. A particular feature of this solution which is the counterpart of the asymptotic flatness of the Schwarzschild solution is that the cylinder shown in $\left(a_{1}\right)$ has constant circumference as $r \rightarrow \infty$. Surface $\left(a_{2}\right)$ corresponds to another global solution with the signature $(+-++)$. The surface $\mathbb{V}$ is topologically a disc 


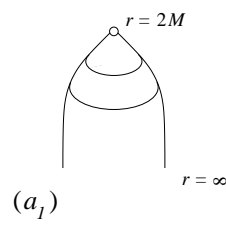

$\left(a_{1}\right)$

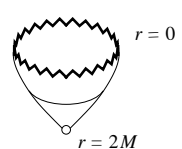

$\left(a_{2}\right)$

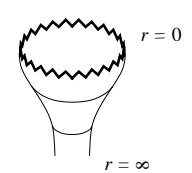

(b)

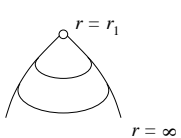

$\left(c_{1}\right)$

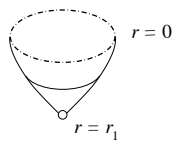

$\left(c_{2}\right)$

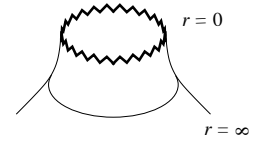

(d)

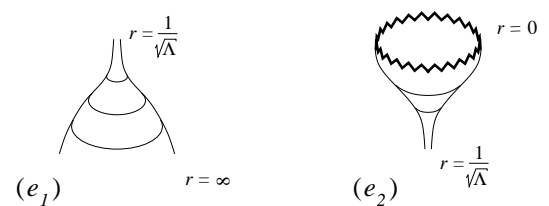

$\left(e_{1}\right)$

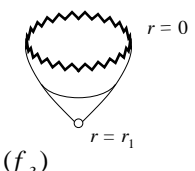

$\left(f_{3}\right)$

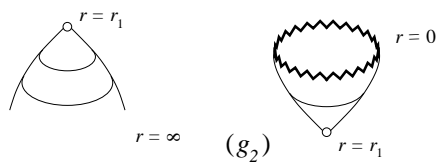

Figure 4: Hyperbolic solutions for $K=-1$. The hollow circles denote possible conical singularities.

with singular curvature at the boundary $r=0$ and, possibly, a conical singularity at the center. Hence the three-dimensional space is a cosmic string surrounded by a cylindrical domain wall of singular curvature. The singular boundary cannot be drawn adequately because it lies at a finite distance but has infinite circumference.

\subsection{3 $\Lambda=0, M<0$}

Here the surface $\mathbb{V}$ is a half plane $r \in(0, \infty), \sigma \in(-\infty, \infty)$ with negative definite metric. The singularity lies along the line $r=0$. From the four-dimensional point of view it represents an infinite planar domain wall of singular curvature located at a finite distance which lives forever.

One may also identify the points $\sigma$ and $\sigma+L$. The resulting surface is shown in Fig. $4(b)$. The corresponding three-dimensional space represents a cylindrical domain wall.

\subsubsection{De Sitter solution $\Lambda>0, M=0$}

This is one more version of the de Sitter solution. There are two surfaces shown in Fig. $4\left(c_{1,2}\right)$ with positive and negative definite metrics, respectively. They are obtained after identifying $\sigma$ with $\sigma+L$. The de Sitter solution corresponds to their universal covering spaces. The surface $\left(c_{2}\right)$ has no singularity at $r=0$ but is incomplete due to the coordinate choice. The present form shows how the de Sitter solution can be deformed to obtain a conical singularity at $r_{1}=\sqrt{3 / \Lambda}$. 


\subsubsection{Anti de Sitter solution $\Lambda<0, M=0$}

In the anti de Sitter case the $\mathbb{V}$ surface is a half plane $r \in(0, \infty), \sigma \in(-\infty, \infty)$ with negative definite metric. It has no singularity but is incomplete at $r=0$.

\subsection{6 $\Lambda>0,3 M>\frac{1}{\sqrt{\Lambda}}$}

The surface $\mathbb{V}$ is the half plane $r>0$ with positive definite metric and a curvature singularity at $r=0$. It describes a planar domain wall. Compactification of $\sigma$ yields the exterior region of a circular domain wall shown in Fig. $4(d)$.

\subsection{7 $\Lambda>0,3 M=\frac{1}{\sqrt{\Lambda}}$}

For a double root horizon two surfaces shown in Fig. $4\left(e_{1,2}\right)$ are obtained, both with negative definite metric. The surface $\left(e_{1}\right)$ is not simply connected. Topologically it is a plane with its centre removed to infinity. The surface $\left(e_{2}\right)$ corresponds to a cylindrical domain wall of singular curvature. The axis of the cylinder is located at an infinite distance.

\subsection{8 $\Lambda>0,0<3 M<\frac{1}{\sqrt{\Lambda}}$}

Due to the two zeros $r_{1,2}$ we obtain three maximally extended solutions, Fig. 4 $\left(f_{1-3}\right)$. The surfaces $\left(f_{1}\right)$ and $\left(f_{3}\right)$ were qualitatively described above but the surface $\left(f_{2}\right)$ is new. It is compact and may contain two conical singularities. An algebraic analysis of the system of equations $N\left(r_{1}\right)=N\left(r_{2}\right)=0$ and $N^{\prime}\left(r_{1}\right)=$ $-N^{\prime}\left(r_{2}\right)$ with $r_{1} \neq r_{2}$ shows that it has no positive roots. Therefore, by fitting appropriately the period $L$ of compactification we can avoid one of them but not both simultaneously, so there exists necessarily a cosmic string. Topologically, the space is a product of a sphere with a line where the sphere must contain at least one conical singularity.

\subsection{9 $\Lambda>0, M<0$}

The function $N$ has one zero so we obtain two Euclidean surfaces. The first $\left(g_{1}\right)$ has the same form as $\left(c_{1}\right)$, and the solution describes an infinite cosmic string without curvature singularity. The second surface $\left(g_{2}\right)$ has the form shown in $\left(f_{3}\right)$ but with negative definite metric.

\subsubsection{0 $\Lambda<0, M>0$}

This case has two inextendible surfaces for $\mathbb{V}$ as in the previous case $\left(g_{1,2}\right)$, but the signature of the metric on both surfaces must be changed. 

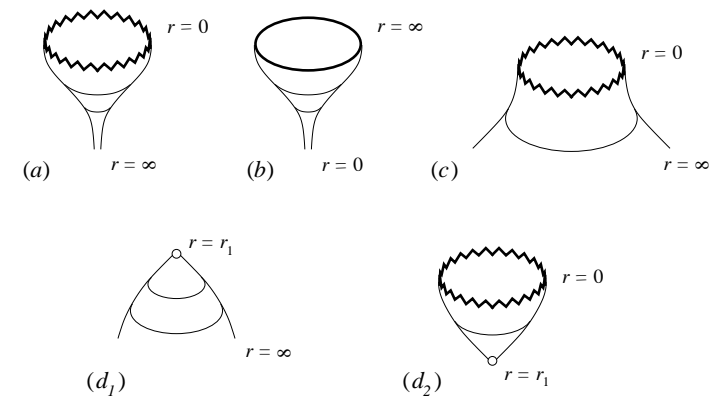

Figure 5: Minkowski plane solutions, $K=0$.

\subsubsection{1 $\Lambda<0, M<0$}

This case is similar to the case $(d) \Lambda>0,3 M>1 / \sqrt{\Lambda}$, but with negative definite metric.

\subsection{Minkowski plane solutions, $K=0$}

For $K=0$ the surface $\mathbb{U}$ is the Minkowskian plane, a cylinder, or a torus and some new topologically interesting solutions arise. The corresponding line element in Schwarzschild coordinates is

$$
d s^{2}=r^{2}\left(d t^{2}-d x^{2}\right)-\frac{d r^{2}}{N(r)}-N(r) d \sigma^{2},
$$

where

$$
N(r)=-\frac{2 M}{r}-\frac{1}{3} \Lambda r^{2}
$$

There are four qualitatively different cases:

\subsection{1 $\Lambda=0, M \neq 0$}

In this case we have a surface shown in Fig. $5(a)$ with positive and negative definite metric for $M>0$ and $M<0$, respectively.

\subsection{2 (Anti) de Sitter solution $\Lambda \neq 0, M=0$}

This is one more representation of the (anti) de Sitter solution for positive and negative cosmological constant. The surface $\mathbb{V}$ is shown in Fig. $5(b)$. In this form it is explicitly conformally flat

$$
d s^{2}=\frac{9}{\Lambda^{2} \rho^{2}}\left(d t^{2}-d x^{2}\right)+\frac{3}{\Lambda \rho^{2}}\left(d \rho^{2}+d \sigma^{2}\right) .
$$




\subsection{3 $\Lambda>0, M>0$}

In this case $N$ has no zero, and the surface $\mathbb{V}$ is shown in Fig. $5(c)$. It describes the outside region of a cylindrical domain wall of curvature singularity. For $\Lambda<0$ and $M<0$ one has simply to change the signature of the whole metric.

\subsection{4 $\Lambda>0, M<0$}

With one zero of $N$ the two surfaces shown in Fig. $5\left(d_{1,2}\right)$ are obtained. They describe a cosmic string and a cosmic string surrounded by a domain wall of curvature singularity.

\section{Summary and Outlook}

The analysis of global solutions given in the present paper exhausts all vacuum solutions to the Einstein equations having the form of a warped product of two surfaces. We have shown that the requirement of maximal extension almost uniquely determines their causal structure. The solutions are classified by the values of a cosmological constant, the constant scalar curvature of one of the surfaces, and an integration constant which for the Schwarzschild solution coincides with its mass. Although these solutions have a simple form and were known locally, their global structure is of great physical interest, describing, e.g., cosmic strings, domain walls, cosmic strings surrounded by domain walls, and solutions with closed timelike curves. Our analysis becomes possible thanks to a systematic method to construct maximally extended surfaces which has been developed for $2 d$ gravity models. A similar approach may be used also in the more general problem including matter fields subject to some symmetry restrictions. The problem seems to be solvable at least in the presence of a $U(1)$ gauge field. Although the Carter-Penrose diagrams have been presented here for all cases, we have to defer a systematic discussion of possible special properties of non-radial geodesics. However, in analogy to the Schwarzschild case we do not expect any surprises regarding the extendability of $4 d$ solutions.

\section{Acknowledgement}

We thank H. Balasin and I. Volovich for discussions. This work has been supported by the Austrian Fonds zur Förderung der wissenschaftlichen Forschung (FWF) Project No. P 10221-PHY. One of the authors (M. K.) thanks the Austrian Academy of Sciences, the Erwin Schrödinger International Institute for Mathematical Physics, the Technical University of Vienna, and the Russian Foundation for Basic Research, Grants RFBR-96-010-0312 and RFBR-96-15-96131, for financial support. 


\section{References}

[1] D. Kramer, H. Stephani, M. MacCallum, and E. Herlt, Exact Solutions of Einstein's Field Equations (Cambridge University Press, Cambridge, 1980).

[2] P. Thomi, B. Isaak, and P. Hajicek, Phys. Rev. D 30, 1168 (1984); P. Hajicek, em ibid. 30, 1178 (1984).

[3] M. O. Katanaev and I. V. Volovich, Phys. Lett. B 175, 413 (1986).

[4] G. Mandal, A. Sengupta, and S. R. Wadia, Mod. Phys. Lett. A 6, 1685 (1991); S. Elitzur, A. Forge, and E. Rabinovici, Nucl. Phys. B359, 581 (1991); E. Witten, Phys. Rev. D 44, 314 (1991); C. G. Callan, S. B. Giddings, J. A. Harvey, and A. Strominger, Phys. Rev. D 45, 1005 (1992); J. Russo, L. Susskind and L. Thorlacius, Phys. Lett. B 292, 13 (1992); T. Banks, A. Dabholkar, M. Douglas and M. O'Laughlin, Phys. Rev. D 45, 3607 (1992); S. P. deAlwis, Phys. Lett. B 289, 278 (1992).

[5] M. O. Katanaev, W. Kummer, and H. Liebl, Nucl. Phys. B486, 353 (1997).

[6] D. Louis-Martinez, J. Gegenberg, and G. Kunstatter, Phys. Lett. B 321, 193 (1994); Phys. Rev. D 51, 1781 (1995); W. M. Seiler and R.W. Tucker, Phys. Rev. D 53, 4366 (1996); A. Barvinsky and G. Kunstatter, Phys. Lett. B 389, 231 (1996).

[7] M.O. Katanaev, W. Kummer, and H. Liebl, Phys. Rev. D 53, 5609 (1996).

[8] W. Kummer and D. J. Schwarz, Nucl. Phys. B382, 171 (1992); F. Haider and W. Kummer, Int. J. Mod. Phys. A 9, 207 (1994); W. Kummer, H. Liebl and D. Vassilevich, Nucl. Phys. B513, 723 (1998).

[9] M. D. Kruskal, Phys. Rev. 119, 1743 (1960); G. Szekeres, Publ. Mat. Debrecen 7, 285 (1960).

[10] B. Carter, "Black hole equilibrium states", in Black holes, ed. C. DeWitt and B. S. DeWitt (New York: Gordon \& Breach, 1973).

[11] M. O. Katanaev, J. Math. Phys. 34, 700 (1993).

[12] T. Klösch and T. Strobl, Class. Quantum Grav. 13, 965 (1996), ibid. 13, 2395 (1996), ibid. 13, 1191 (1996), ibid. 14, 1689 (1997).

[13] M. O. Katanaev, J. Math. Phys. 38, 946 (1997).

[14] J. Carot and J. da Costa, Class. Quantum Grav. 10, 461 (1993); H.-J. Schmidt, Grav. Cosmol. 3, 185 (1997).

[15] A. Z. Petrov, Einstein Spaces (Pergamon Press, Oxford - London, 1969). 
[16] C.-g. Huang and C.-b. Liang, Phys. Lett. A 201, 27 (1995); J. P. S. Lemos, Phys. Lett. B 353, 46 (1995); D. R. Brill, J. Louko, and P. Peldán, Phys. Rev. D 56, 3600 (1997).

[17] R.-G. Cai and Y.-Z. Zhang, Phys. Rev. D 54, 4891 (1996); W. L. Smith and R. B. Mann, Phys. Rev. D 56, 4942 (1997); L. Vanzo, Phys. Rev. D 56, 6475 (1997); R.-G. Cai, J.Y. Ji, and K.-S. Soh, Phys. Rev. D 57, 6547 (1998).

[18] M. O. Katanaev, J. Math. Phys. 31, 882 (1990).

[19] L. D. Landau and E. M. Lifshitz, The Classical Theory of Fields (Pergamon, New York, 1962).

[20] F. Kottler, Ann. Phys. (Leipzig) 56, 401 (1918).

[21] W. Israel, Phys. Rev. 164, 1776 (1967).

[22] M. Heusler, Black Hole Uniqueness Theorems (Cambridge U.P., Cambridge, 1996). 